\title{
Digital Watermarking Algorithm for Embedding Color Image using Two Level DWT
}

\author{
Maneesha Paliwal \\ Research scholar \\ Computer Science and Engineering Department \\ Samrat Ashok Technological Institute Vidisha
}

(M.P.),

\begin{abstract}
Digital watermarking is an important technique that deals with information hiding which is used to hide proprietary information in digital media like photographs, digital music, or digital video. In this paper we present a technique on digital watermarking for real time application. We have implemented the digital watermarking on image by applying two level Discrete Wavelet Transform by which we embed watermark and for extraction apply Inverse DWT transform. The performance of image is calculated in terms of PSNR \& MSE.
\end{abstract}

\section{General Terms}

Digital watermarking, Discrete Wavelet Transform.

\section{Keywords}

DWT, DCT, PSNR, MSE.

\section{INTRODUCTION}

The development of effective digital image copyright protection methods have recently become an important and necessary requirement in the multimedia industry due to the ever-increasing unauthorized manipulation and reproduction of original digital objects. The new technology of digital watermarking has been advocated by many researchers as the best method to such multimedia copyright protection problem [1]. It is expected that digital watermarking would have a wide-range of practical applications in digital cameras, medical imaging, image databases, and video-on-demand systems etc.

Digital watermarking deals with information hiding which is used to hide proprietary information in digital media like photographs, digital music, or digital video [2-3]. The ease with which digital content can be exchanged over the Internet has created copyright infringement issues. Copyrighted material can be easily exchanged over peer-to-peer networks, and it has caused major concerns to content providers engaged

in producing the contents. For an efficient watermarking method, it should be robust to compression, filtering, rotation, scaling cropping, and collusion attacks among many other digital processing operations. The existing digital image watermarking techniques can be grouped into two major classes' namely Spatial Domain Watermarking and Transform Domain Watermarking techniques. In comparison with spatial domain techniques [4], transform-domain watermarking techniques (DWT) are generally more effective in terms of the imperceptibility and robustness requirements of digital watermarking algorithms [5-6]. A transform domain technique is proposed which shows greater robustness to common signal distortions. The main advantage of the proposed wavelet-based technique lies in the method used to embed the watermark in low frequency band using blending technique. Performance improvements in DWT-based digital

\author{
Yogendra Kumar Jain, Ph.D \\ Head of Department \\ Computer Science and Engineering Department \\ Samrat Ashok Technological Institute Vidisha \\ (M.P.),
}

image watermarking algorithms could be obtained by increasing the level of DWT.

\section{RELATED WORK}

Su et.al, introduced a blind digital watermarking algorithm for embedding color image into color image[6]. The two-level DCT is introduced and used to embed color watermark image into color host image, which is different with the traditional DCT and reducing the redundancy of watermark information after two-level DCT, nine AC coefficients in different positions of each sub-block are selected and quantified to embed watermark information. Moreover, only the extraction rules are used to extract watermark from the watermarked image without resorting to the original host image or watermark image.

Mistry introduced digital watermarking methods- Spatial domain (like LSB) and transform domain (like DCT, DWT) methods[7]. The spatial domain is the normal image space, in which a change in position in image directly projects to a change in position in space. For example Least Significant bit (LSB) method. Transform Domain method produce high quality watermarked image by first transforming the original image into the frequency domain by the use of Fourier Transform, Discrete Cosine Transform (DCT) or Discrete Wavelet transforms (DWT). It was observed that transform watermarking is comparatively much better than the spatial domain encoding. Transform based methods are very efficient and having more robustness for image processing attacks.

Zhang and Yong Ping proposed a novel watermarking algorithm which uses features of lower frequency sub-band of wavelet coefficient to limit the positions for watermarking[8]. The positions are taken as indexes of points to embed watermarking and may these positions to medium frequency space to carry watermarking.

Thongkor et al., presents image watermarking based on DWT coefficients modification for social networking services [9]. The decomposition is done on the blue component of original host image by the DWT to obtain the coefficients in LL subband, and some of them are used to carry watermark signal.

Deb et al., proposed a combined DWT and discrete cosine transform based digital image watermarking technique for copyright protection[10]. The watermarking bits are embedded in the low frequency band of each DWT block of selected discrete wavelet transform sub-band.

Sridhar B and Arun C proposed secure multiple image watermarking techniques using DWT with the motivation to maintain the quality of the image in which the original image was interlaced into even and odd rows of images and deinterlaces the two images[11]. Wavelet based approach is employed for hiding watermark images. 


\section{WATERMARKING TECHNIQUES}

(A) Spatial Domain: The most basic method for embedding the watermark in spatial domain is to add pseudo random noise pattern to the intensity of image pixels. The noise signal is usually integers like $(-1,0,1)$ or sometimes floating point numbers [12].

(a) Least Significant Bit Modification: A digital image version of this analogue image contains sampled values of the function at discrete locations and pixels. These values are representation of the image in the spatial domain or often referred to as the pixel domain. Spatial embedding inserts message into the image pixels [12].

(B) Transform Domain: Transform domain embeds a message by modifying the transform coefficients of the cover message as opposed to thepixel values. Ideally, transform domain has the effect in the spatial domain of apportioning the hidden information through different order bits in a manner that is called robust. There are many numbers of transforms that can be applied in digital images, but there are especially three most commonly used in image watermarking. They are Discrete Cosine Transform (DCT), Discrete Fourier Transform(DFT), ) and DiscreteWavelet Transform (DWT) [12].

(a) Discrete Fourier Transform: Fourier Transform (FT) is an operation that transforms a continuous function into its frequency components. Theequivalent transform for discrete valued function requires the Discrete Fourier Transform (DFT). In digital image processing, the even functions that are not periodic can be expressed as the integral of sine and cosine multiplied by a weighing function. This weighing function makes the coefficients of the Fourier Transform of the signal. FourierTransform allows analysis and processing of the signal in its frequency domain by means of analyzing and modifying these coefficients [12].

(b) Discrete Cosine Transform: Discrete Cosine Transform is correlated to DFT in a sense that it transforms a time domain signal into its frequency components. The DCT though only uses the real parts of the DFT coefficients. In the form of property, the DCT has a strong energy compaction property and most of the signal information tends to be concentrated in a few low-frequency components of the DCT. The JPEG compression technique uses this property for separate and remove insignificant high frequency components in images [12].

(c) Discrete Wavelet Transform: Wavelet Transform is a recent technique frequently used in digital image compression, processing, watermarking etc. The transforms are based on small waves, called wavelet, of unreliable frequency and limited duration. A wavelet series is a representation of a square integral function by a certain ortho-normal series generated by a wavelet. Besides, the properties of wavelet could decompose original signal into wavelet transform coefficients which contains the position information. The original signal can be completely reconstructed by performing Inverse Wavelet Transformation of these coefficients. Watermarking in the wavelet transform domain is generally a problem of embedding watermark in the sub bands of the cover image [12].

\section{APPLICATIONS OF DIGITAL WATERMARKING}

(a) Copyright Protection:

Copyright protection is the first targeted application for digital watermarking. In digital multimedia, watermarking techniques is used as copyright security to identify the copyright vendor. The vendor of the digital media can protect his content from being used commercially.

\section{(b) Fingerprinting:}

A key issue in real time application is illegal distribution of copy righted digital content like movies, which can be avoided by watermarking the video using a technique called fingerprinting. Fingerprinting is used to mark out the origin of illegal copies.

\section{(c) Tamper proofing:}

Digital photography authentication has become a great concern as they can be easily tampered. Such problems have hindered the application of digital images for insurance claims, courtroom evidence, journalistic photography and copyright claims.

\section{(d) Medical Image Watermarking:}

The evolution of medical information system, supported by advance in information technology, enables information to be shared between distant health professionals. on the other hand, this could pose a threat to privacy of information if security measures are not considered.

\section{PROPOSED METHODOLOGY Proposed Algorithm}

(1) Consider the image to be resized to $\mathrm{N} \times \mathrm{N}$; (Original image I having $\mathrm{N}$ value of pixel). Select the pixel on which Semi-fragile watermarking needs to apply.

(2) Digital Image is applied for authentication, and then takes the two levels DWT.

(3) Based on given wavelet bands HL2, LH2, and HH2 (Frequency Coordinates), we calculate the image feature matrix $\mathrm{K}$

$K(I, j)=|H L 2(i, j)|+|L H 2(i, j)|+|H H 2(i, j)|$

(4) Above feature matrix $\mathrm{K}$ then stored their value to a pool after calculation of hash value for each sampled single value bands.

(5) Calculate the N Random matrix whose inputs are later pass through LPF (Resized Image).

(6) Using this feature matrix \& mark sequence the process of watermarking is applied by XOR ing the wavelets with mark values.

(7) Obtained watermark having interleaved value vector after each sequence $\mathrm{W}$ must be above adaptive threshold value $\mathrm{T}$. Watermark value is

if $\mathrm{W}(\mathrm{i}, \mathrm{j})<\mathrm{T} ; \mathrm{i}=1$ ( $\mathrm{i}$ is a selected block value for an image, $\mathrm{j}$ is feature extracted)

if $\mathrm{W}(\mathrm{i}, \mathrm{j})>\mathrm{T} ; \mathrm{i}=0$ (Interleaving generates a new key

(8) The image features are factorized using DWT and the generated coefficient matrix is quantized to produce the image hash. 
(9) For this purpose, first we generate a pseudorandom sequence using extracted feature \& mark sequence.

$$
X_{K+1}=\mu X_{K}\left(1-X_{K}\right) \text {, where } 1<\mu=<4 \text {. }
$$

(10) Finally this mark value \& hash value is XORed to get the image Watermarked in semi fragile manner. Thus in future the practical implementation of design concepts will make the things clear about the result. Work needs to insert an imaginary type of noise to create distortion.

(11) Result will be calculated in term of PSNR values.

\section{Proposed Technique:}

1. In proposed work we start by selecting two kinds of image one is for cover image and another is for watermark image.

2. Here we apply 2 level DWT transform i.e they combine the cover image and watermark image.

3. Now a watermarked image is created.

4. Apply Inverse level DWT transform to extract watermark image.

5. Calculate PSNR \& MSE value for Embedding Process.

6. Calculate PSNR \& MSE value for Extraction Process

\section{RESULT METHODOLOGY}

\section{The metrics used are}

1. Mean Square Error (MSE): Mean Square Error (MSE) for two $\mathrm{P} \times \mathrm{Q}$ monochrome images $(\mathrm{G}$ and $\mathrm{R}$ ) where one of the images is considered a noisy approximation of the other is defined as:

$$
\operatorname{MSE}(x)=\frac{ \pm}{N}\left\|x-x^{\wedge}\right\|^{2}=\frac{1}{N} \sum_{i=1}^{N}\left(x-x^{\wedge}\right)^{2}
$$

2. Peak Signal to Noise Ratio (PSNR): The

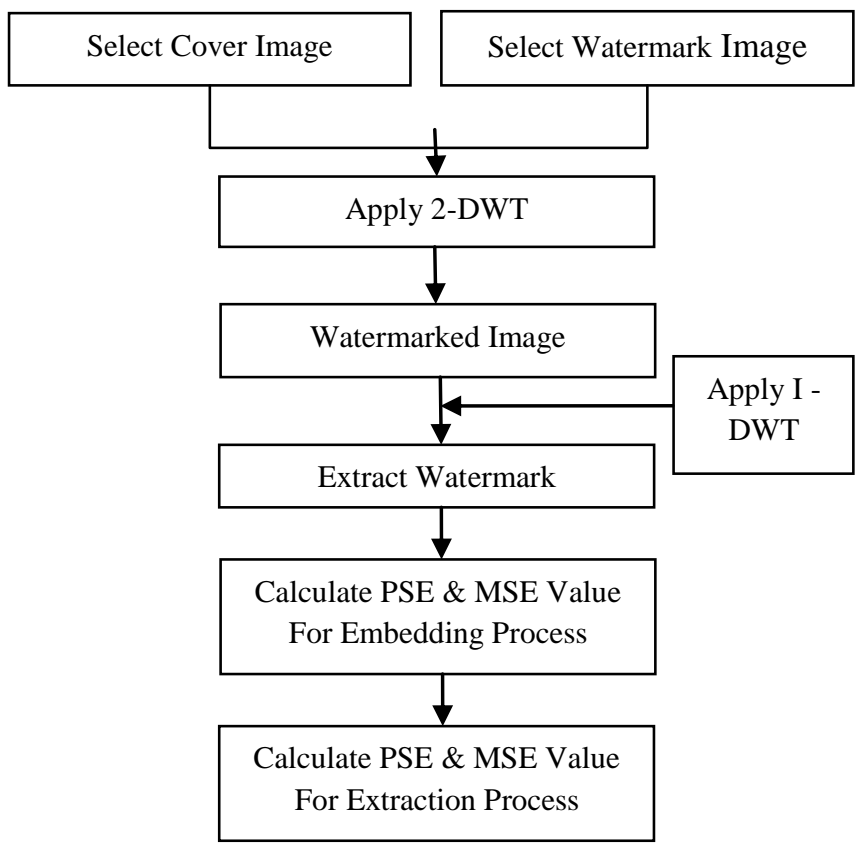

Fig. 1 Flow Chart of Proposed Work

PSNR is most commonly used as a measure of the quality of de-speckled image. The PSNR is defined as:

$$
\text { PSNR }=10 \log 10\left(\frac{M A X\left(x^{2}\right)}{\operatorname{MSE}(x)}\right.
$$

Figure 2 shows the original cover image, Figure 3 shows the original watermarked image, Figure 4 shows the original watermark and Figure 5 shows the watermark extracted from the watermarked image. All these images represents that the watermark can be easily extract from the watermarked image by the proposed work. Results are shows in the Table 1

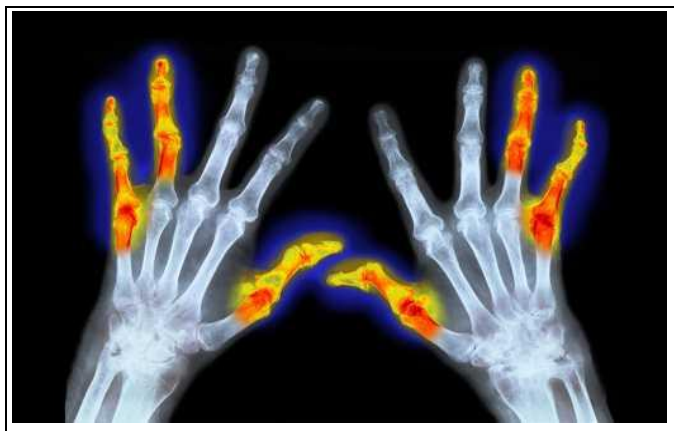

(a)

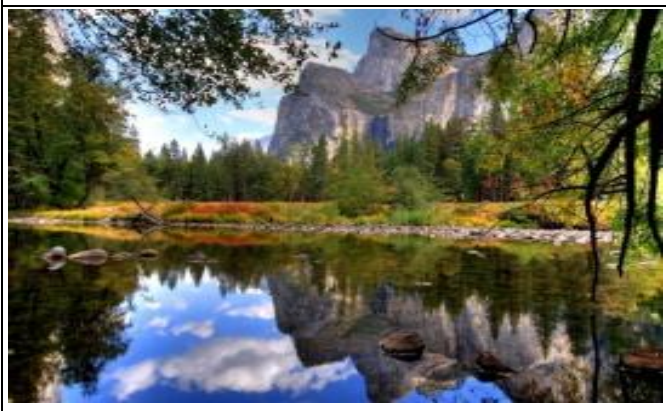

(c)

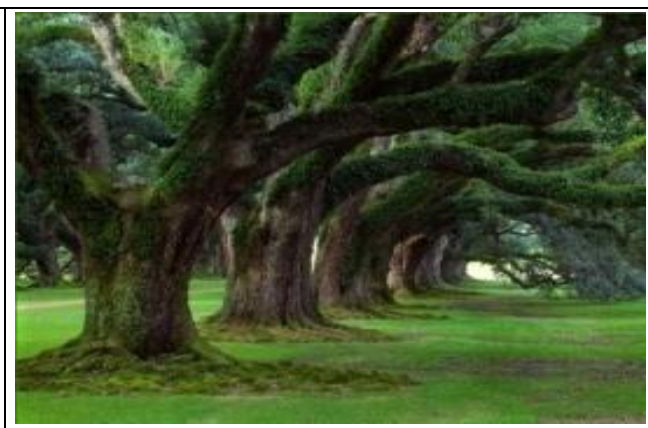

(b)

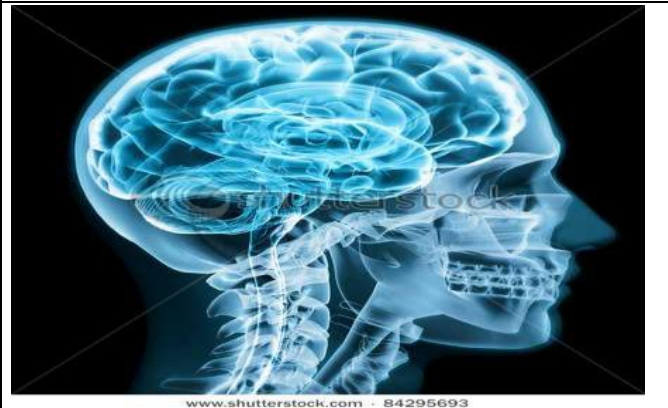

(d) 


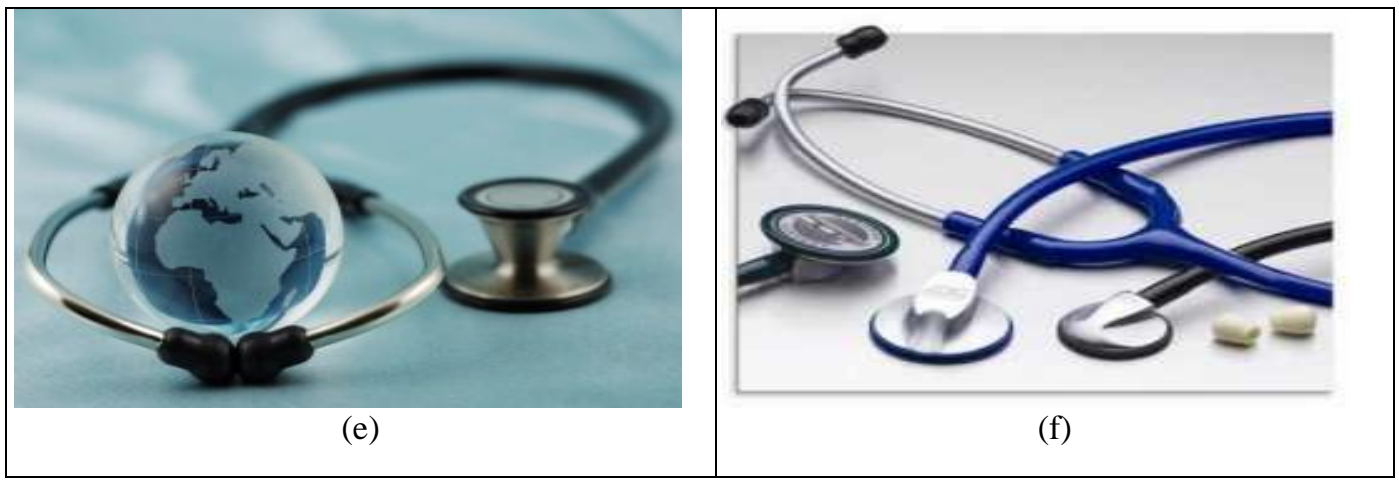

Fig. 2 Original Cover Images

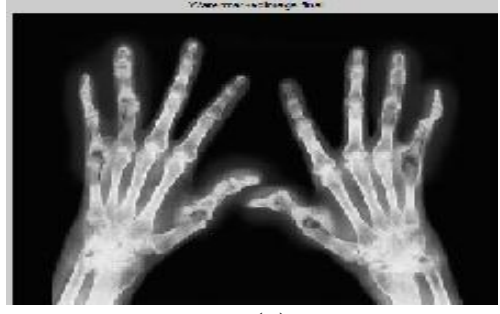

(a)

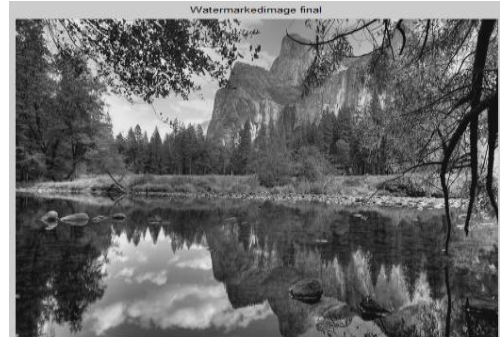

(c)

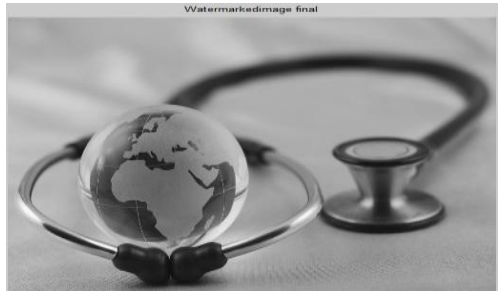

(e)

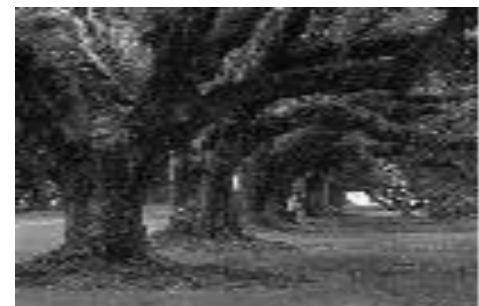

(b)

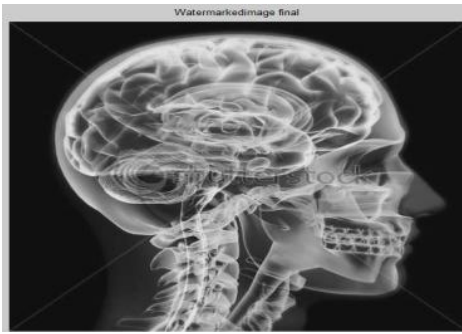

(d)

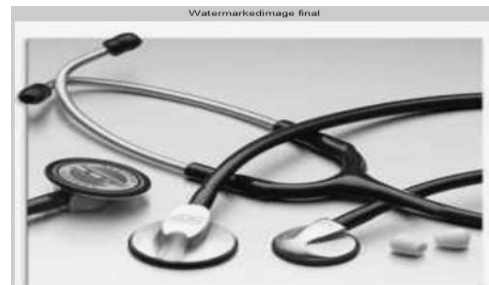

(f)

Fig. 3: Cover Images

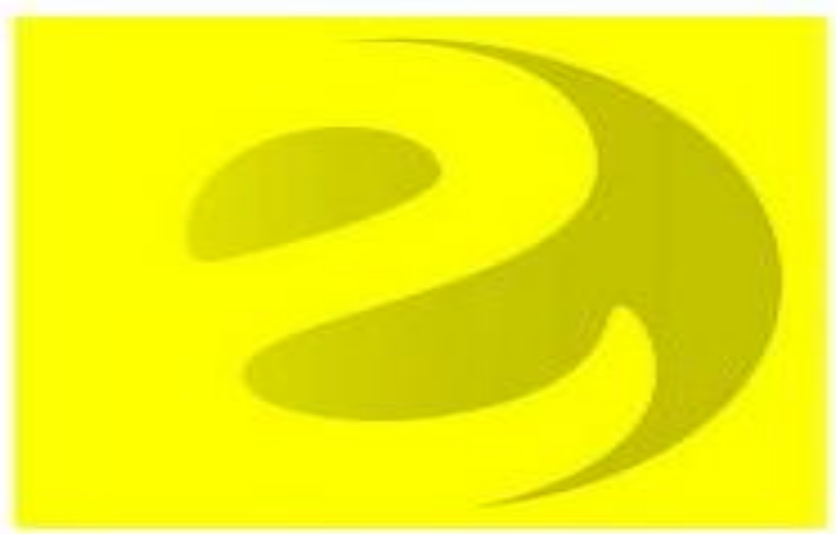

Fig. 4: Original watermark 


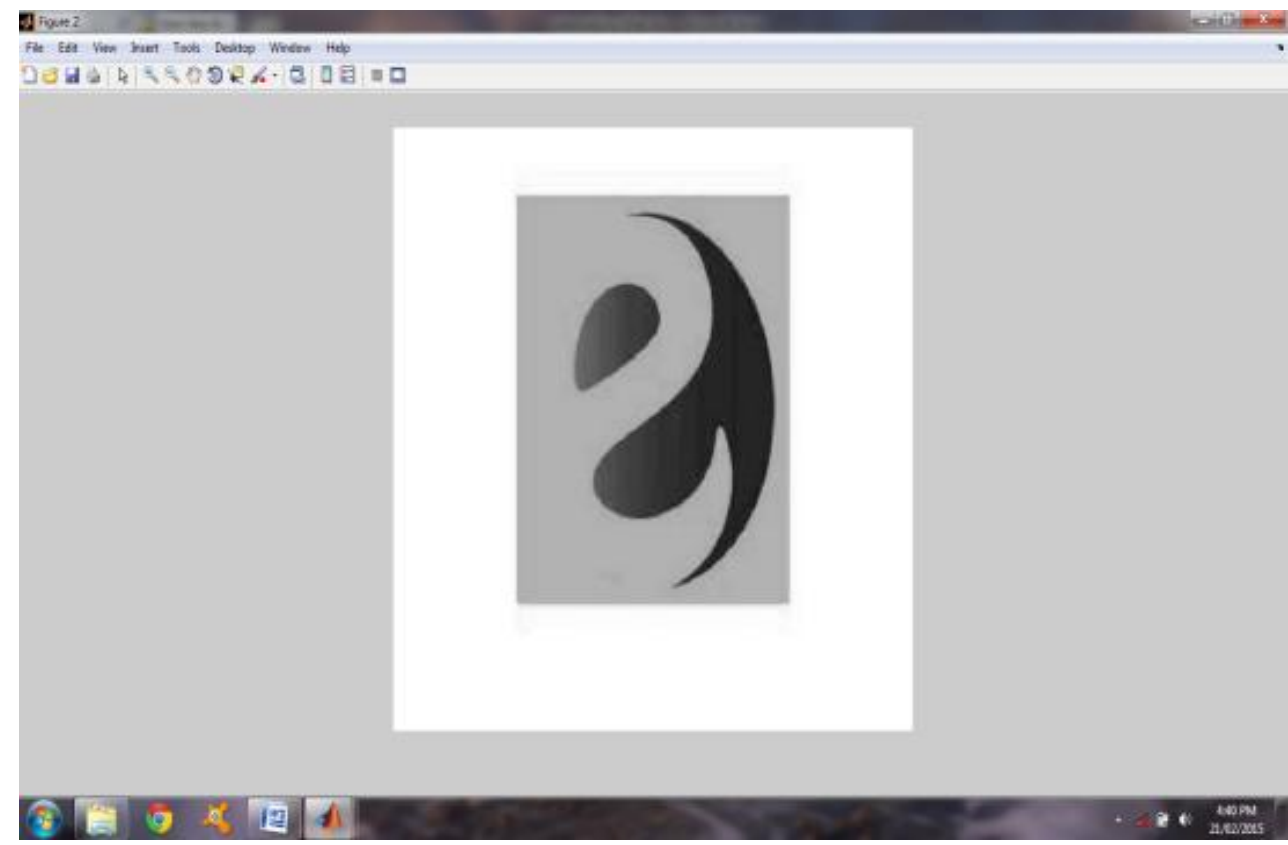

Figure 5 Extracted Watermark

Table 1

\begin{tabular}{|c|c|c|c|c|}
\hline \multirow{2}{*}{ Image } & \multicolumn{2}{|c|}{ Su et.al[6] } & \multicolumn{2}{c|}{ Proposed Method } \\
\cline { 2 - 5 } & PSNR & Execution Time & PSNR & Execution Time \\
\hline (a) & 39.161 & 11.815 & 64.871 & 6.451 \\
\hline (b) & 39.605 & 11.698 & 65.192 & 4.886 \\
\hline (c) & 39.607 & 11.79 & 65.63 & 3.978 \\
\hline (d) & 39.609 & 12.043 & 65.566 & 4.4111 \\
\hline (e) & 39.610 & 11.689 & 65.099 & 5.4550 \\
\hline (f) & 39.610 & 11.974 & 65.099 & 4.1458 \\
\hline
\end{tabular}

In the table 1, PSNR values and Execution times are given. Process of these results is, taken cover image and perform watermark on the cover image, by this process we obtained the watermarked image and calculate the PSNR and MSE values for embedding image. Now extract the watermark and calculate the PSNR and MSE values for extraction.

\section{CONCLUSION}

To calculate results we have used Matlab. From the obtained results we observed that the Performance of proposed method is better in terms of PSNR and Execution times and we found that 2-level DWT method produces better result. For the objective evaluation Execution Time and PSNR have been discussed. The higher PSNR indicates better perceptual quality of image. Hence, more PSNR is always desirable without trade off in image quality. In our work we improved the value of PSNR by reducing the value of Execution Time. It shows that good quality of watermarked image can be obtained by the proposed work.
In future it will improve by combining the proposed technique with other algorithm to achieve better performance and further reduce the Execution Time value in dealing with images and video with more complex background for surveillance and biometric applications.

\section{ACKNOWLEDGMENTS}

I would like to thank Dr. Yogendra Kumar Jain, Head, Department of Computer Science and Engineering who has contributed towards development of the template.

\section{REFERENCES}

[1] Vidyasagar M. Potdar, Song Han, Elizabeth Chang, "A Survey of Digital Image Watermarking Techniques", 3rd International Conference on Industrial Informatics (INDIN), pp. 709-716, 2005.

[2] Ibrahim, R. and Kuan, T. S., "Steganography Imaging (SIS): Hiding Secret Message inside an Image", Proceedings of the World Congress on Engineering and Computer Science, San Francisco, USA, 2010. 
[3] Nikita Kashyap and Sinha G.R., "Image watermarking using 2-level DWTI, Advances in Computational Research", Vol. 4, Issue 1, pp.42-45, 2012.

[4] L. Robert, T. Shanmugapriya, "A Study on Digital Watermarking Techniques", International Journal of Recent Trends in Engineering, 2009.

[5] G. RoslineNesaKumari, B. Vijaya Kumar, L. Sumalatha, and Dr V. V. Krishna, "Secure and Robust Digital Watermarking on Grey Level Images", International Journal of Advanced Science and Technology, 2009.

[6] Su, Q., Niu, Y., Liu, X., \& Yao, T. 'A novel blind digital watermarking algorithm for embedding color image into color image". Optik-International Journal for Light and Electron Optics, pp.3254-3259, 2013.

[7] Darshana Mistry, "Comparison of Digital Watermarking methods", 21st Computer Science Seminar SA1-T1-7, IJCSE, 2010.

[8] Zhang and Yong Ping, "Digital Watermarking Technique for Images based on DWT", Proceedings of
Seventh IEEE International Conference on Digital Object Identifier, pp. 1-4, 2011.

[9] Thongkor K, Mettripum N, Promoun T and Amornraksa T, "Image Watermarking based on DWT Coefficients Modification for Social Networking Services", Proceedings of Tenth IEEE International Conference on Digital Object Identifier, pp. 1-6, 2013.

[10] Deb K, Alseraj, Hoque and Sarkar, "Combined DWTDCT based Digital Image Watermarking Technique for Copyright Protection", Proceedings of Seventh IEEE International Conference on Digital Object Identifier, pp. 458-461, 2012.

[11] Sridhar B and Arun C, "On Secure Multiple Image Watermarking Technique using DWT", Proceedings of Third IEEE International Conference on Digital Object Identifier, pp. 1-4, 2012.

[12] Bhupendra Ram, Member, IEEE "Digital Image Watermarking Technique Using Discrete Wavelet Transform And Discrete Cosine Transform" International Journal of Advancements in Research \& Technology, Volume 2, Issue 4, 2013. 